\title{
High-dose tranexamic acid reduces intraoperative and postoperative blood loss in posterior lumbar interbody fusion
}

\author{
Junichi Kushioka, MD, ${ }^{1}$ Tomoya Yamashita, MD, ${ }^{2}$ Shinya Okuda, MD, PhD, ${ }^{2}$ \\ Takafumi Maeno, MD, PhD, ${ }^{2}$ Tomiya Matsumoto, MD, PhD, ${ }^{2}$ Ryoji Yamasaki, MD, PhD, ${ }^{3}$ and \\ Motoki Iwasaki, MD, PhD² \\ ${ }^{1}$ Department of Orthopaedic Surgery, Osaka University Graduate School of Medicine; 'Department of Orthopaedic Surgery, \\ Osaka Rosai Hospital; and ${ }^{3}$ Department of Orthopaedic Surgery, Osaka Police Hospital, Osaka, Japan
}

\begin{abstract}
OBJECTIVE Tranexamic acid (TXA), a synthetic antifibrinolytic drug, has been reported to reduce blood loss in orthopedic surgery, but there have been few reports of its use in spine surgery. Previous studies included limitations in terms of different TXA dose regimens, different levels and numbers of fused segments, and different surgical techniques. Therefore, the authors decided to strictly limit TXA dose regimens, surgical techniques, and fused segments in this study. There have been no reports of using TXA for prevention of intraoperative and postoperative blood loss in posterior lumbar interbody fusion (PLIF). The purpose of the study was to evaluate the efficacy of high-dose TXA in reducing blood loss and its safety during single-level PLIF.

METHODS The study was a nonrandomized, case-controlled trial. Sixty consecutive patients underwent single-level PLIF at a single institution. The first 30 patients did not receive TXA. The next 30 patients received $2000 \mathrm{mg}$ of intravenous TXA 15 minutes before the skin incision was performed and received the same dose again 16 hours after the surgery. Intra- and postoperative blood loss was compared between the groups.

RESULTS There were no statistically significant differences in preoperative parameters of age, sex, body mass index, preoperative diagnosis, or operating time. The TXA group experienced significantly less intraoperative blood loss (mean $253 \mathrm{ml}$ ) compared with the control group (mean $415 \mathrm{ml} ; p<0.01$ ). The TXA group also had significantly less postoperative blood loss over 40 hours (mean $321 \mathrm{ml}$ ) compared with the control group (mean $668 \mathrm{ml} ; \mathrm{p}<0.01$ ). Total blood loss in the TXA group (mean $574 \mathrm{ml}$ ) was significantly lower than in the control group (mean $1080 \mathrm{ml} ; p<0.01$ ). From 2 hours to 40 hours, postoperative blood loss in the TXA group was consistently significantly lower. There were no perioperative complications, including thromboembolic events.
\end{abstract}

CONCLUSIONS High-dose TXA significantly reduced both intra- and postoperative blood loss without causing any complications during or after single-level PLIF.

https://thejns.org/doi/abs/10.3171/2016.8.SPINE16528

KEY WORDS tranexamic acid; perioperative blood loss; posterior lumbar interbody fusion; spine surgery; antifibrinolytic drug; lumbar degenerative disease; surgical technique

$\mathrm{P}$ OSTERIOR lumbar interbody fusion (PLIF) has resulted in satisfactory outcomes for the treatment of unstable lumbar degenerative disorders. In the last decade the number of patients who underwent PLIF significantly increased. ${ }^{911}$ Conventional PLIF is not a minimally invasive procedure, and it is important to carefully monitor blood loss, especially from the epidural space. An increase in intraoperative blood loss may lead to hypoten- sion, disturbance in oxygenation of organs, and difficulty visualizing the operative field for surgeons. Postoperative blood loss may increase the formation of epidural hematomas that cause neurological disorders, coagulopathy, and anemia, which delay postoperative recovery and prolong patient hospitalization.

Fibrinolysis transiently increases during surgery, ${ }^{4}$ and increased fibrinolysis contributes to perioperative blood

ABBREVIATIONS aPTT = activated partial thromboplastin time; $\mathrm{BMI}=$ body mass index; $\mathrm{Hb}$ = hemoglobin; PLIF = posterior lumbar interbody fusion; PLT = platelet; PT-INR = prothrombin time-international normalized ratio; TXA = tranexamic acid.

SUBMITTED May 9, 2016. ACCEPTED August 31, 2016.

INCLUDE WHEN CITING Published online November 25, 2016; DOI: 10.3171/2016.8.SPINE16528. 
loss during spine surgery. ${ }^{7}$ Tranexamic acid (TXA; Daiichi Sankyo Co.) is a synthetic antifibrinolytic drug that competitively blocks the lysine binding sites of plasminogen, plasmin, and tissue plasminogen activator. TXA can delay fibrinolysis and blood clot degradation. ${ }^{5}$ Several reports have described the use of TXA in spine surgery without an increase in the risk of postoperative complications, including thromboembolism. ${ }^{12,13}$ However, previous studies had limitations in terms of different TXA dose regimens, different levels and numbers of fused segments, and different surgical techniques. Therefore, in this study, TXA dose regimens, surgical techniques, and fused segments were strictly limited. The purpose of this study was to evaluate the efficacy of high-dose TXA in reducing blood loss and its safety during single-level PLIF.

\section{Methods}

\section{Study Design and Patient Population}

This study was a nonrandomized case-controlled trial. We evaluated 60 consecutive patients who had experienced lumbar degenerative disease and had failed conservative treatment between April 2013 and March 2015. All patients underwent single-level PLIF. The patients were divided into 2 groups based on whether they received TXA or not. Between April 2013 and June 2014, the procedure without TXA was performed on 30 patients (control group), and between July 2014 and March 2015, the procedure with TXA was conducted on another group of 30 patients (TXA group). This study was approved by the research ethics committee of Osaka Rosai Hospital.

Patients with chronic renal failure, cirrhosis of the liver, chronic heart failure, allergy to TXA, history of thromboembolic disease, bleeding disorders, hypercoagulation status, disseminated intravascular coagulation, those undergoing revision surgery, and those receiving antiplatelet and/or anticoagulant drugs were excluded.

\section{Drug Dose}

A dose of $2000 \mathrm{mg}$ of TXA was given as a single-bolus loading dose intravenously over 15 minutes before the skin incision was made. The same single-bolus dose was given again 16 hours after the surgery. It was reported that the plasma concentration of TXA was maintained during the first 16 hours after administration, although the blood half-life of TXA is reported to be 2 hours. ${ }^{10}$

\section{Surgical Technique}

All procedures were performed by 5 certified orthopedic spine surgeons who had more than 10 years of experience with the timing of PLIF. All PLIF procedures were performed using the same technique, as described elsewhere. ${ }^{8}$ Briefly, PLIF was performed using the pedicle screw system with Expedium (Depuy Acromed) and local bone grafting with Brantigan interbody fusion cages (Depuy Acromed). Bilateral total facetectomy was performed to prevent excessive retraction of the neural elements during discectomy and bone grafting and to achieve an extensive bone graft area and a large amount of bone graft in the disc space. ${ }^{8}$ In all cases, autografting was performed using local laminae. The posterior iliac crest was
TABLE 1. Patient demographics

\begin{tabular}{|c|c|c|c|}
\hline Variable & $\begin{array}{l}\text { Control } \\
\text { Group }\end{array}$ & $\begin{array}{l}\text { TXA } \\
\text { Group }\end{array}$ & $\begin{array}{c}p \\
\text { Value }\end{array}$ \\
\hline No. of patients & 30 & 30 & \\
\hline Mean age $\pm S D$ (yrs) & $71.5 \pm 7.0$ & $67.8 \pm 12.0$ & $0.15^{\star}$ \\
\hline $\mathrm{M}: \mathrm{F}$ & $14: 16$ & $15: 15$ & $0.50 \dagger$ \\
\hline Mean BMI \pm SD & $24.3 \pm 3.2$ & $23.3 \pm 2.8$ & $0.19 \ddagger$ \\
\hline Diagnosis & & & $0.69 \S$ \\
\hline Degenerative spondylolisthesis & 25 & 22 & \\
\hline Isthmic spondylolisthesis & 2 & 5 & \\
\hline Disc herniation & 2 & 2 & \\
\hline Foraminal stenosis & 1 & 1 & \\
\hline $\begin{array}{l}\text { * Welch's t-test. } \\
\text { † Fisher's exact test. } \\
\text { † Student t-test. } \\
\text { § Chi-square test of independence. }\end{array}$ & & & \\
\hline
\end{tabular}

not harvested, and posterolateral arthrodesis was not performed at any level. An intraoperative blood cell salvage system was used, and all autologous blood was transfused during the surgery. A negative-pressure suction drain (3.5$\mathrm{mm}$ SB Vac; Sumitomo Bakelite) was placed. The drain was routinely removed 40 hours after the operation.

All patients wore compression stockings on both legs during bed rest, and a foot pump was used for both legs. No patient received chemoprophylaxis against venous thromboembolism during or after surgery. Hypotensive anesthesia techniques were not used in this study. Nonsteroidal antiinflammatory medication was discontinued at least 24 hours before surgery. ${ }^{2,11}$

\section{Assessment and Statistical Analysis}

Postoperative blood loss was calculated every 2 hours for 40 hours. Total blood loss was calculated as the sum of intraoperative and postoperative blood loss. Blood tests were routinely performed preoperatively and on the first postoperative day. Hemoglobin (Hb), platelets (PLTs), prothrombin time-international normalized ratio (PT-INR), and activated partial thromboplastin time (aPTT) were evaluated. Operative time was also measured and perioperative complications were surveyed. Statistical differences between the two groups were compared using the Student t-test, Welch's t-test, or the Mann-Whitney U-test for continuous variables and the chi-square test of independence or Fisher's exact probability test for categorical variables. A p value $<0.05$ was considered statistically significant.

\section{Results}

The patient characteristics over the study periods are shown in Table 1. There were no statistical differences in preoperative parameters of age, sex, body mass index (BMI), or diagnosis. The preoperative Hb, PLT, PT-INR, and aPTT were not significantly different between the groups (Table 2). Significant differences were not noted in the operation time between the groups. The TXA group (mean $253 \mathrm{ml}$ ) had significantly less intraoperative blood 
TABLE 2. Preoperative hematological data

\begin{tabular}{lclc}
\hline Parameter & Control Group & TXA Group & p Value \\
\hline $\mathrm{Hb}(\mathrm{g} / \mathrm{dl})$ & $13.3 \pm 1.6$ & $13.3 \pm 1.3$ & $0.86^{*}$ \\
\hline $\mathrm{PLTs}\left(10^{9} / \mathrm{L}\right)$ & $21.1 \pm 4.8$ & $23.0 \pm 5.5$ & $0.17^{*}$ \\
\hline PT-INR & $0.96 \pm 0.05$ & $0.98 \pm 0.04$ & $0.08^{*}$ \\
\hline aPTT $(\mathrm{sec})$ & $26.6 \pm 2.9$ & $26.1 \pm 2.0$ & $0.22 \dagger$ \\
\hline
\end{tabular}

Data presented as means \pm standard deviations unless otherwise indicated.

* Student t-test.

† Welch's t-test.

loss compared with the control group (mean $415 \mathrm{ml}$, p < 0.01; Table 3). Intraoperative blood loss in the TXA group was reduced by $39 \%$ compared with the control group. The TXA group also had significantly less postoperative blood loss over 40 hours (mean $321 \mathrm{ml}$ ) compared with the control group (mean $668 \mathrm{ml}$; $\mathrm{p}<0.01$ ). Postoperative blood loss in the TXA group was reduced by $52 \%$. The total blood loss in the TXA group (mean $574 \mathrm{ml}$ ) was significantly lower than that in the control group (mean 1080 $\mathrm{ml} ; \mathrm{p}<0.01$ ). Total blood loss in the TXA group was reduced by $46 \%$ (Table 3, Fig. 1). From 2 hours to 40 hours, postoperative blood loss in the TXA group was consistently significantly lower than that in the control group (Fig. 2). No obvious difference between surgeons was detected in the operating time and the amount of blood loss.

No patient required allogeneic blood transfusion during or after the surgery in either group. Hb and PLT levels on the first postoperative day were higher in the TXA group than in the control group (Table 4). There were no serious perioperative complications such as the formation of epidural hematoma, deep vein thrombosis, pulmonary embolism, allergic reaction, liver or kidney failure, or cardiopulmonary complications in either group. No minor complications such as headache, nausea, vomiting, or diarrhea associated with the use of TXA were recorded.

\section{Discussion}

The results of this study have revealed that high-dose TXA (2000 mg) decreases both intra- and postoperative blood loss when used during and after single-level PLIF without causing any side effects. TXA has been used widely for many surgeries, and many positive effects on blood loss have been reported, but a concern about the relationship between the use of TXA and thromboembolic events remains unclear. In this study, there were no thromboembolic events with TXA, similar to previous studies. ${ }^{1,6,14}$ It is speculated that administration of TXA did not increase the risk of thromboembolism because TXA reduces blood loss by deactivating the fibrinolytic system, not by activating the coagulation cascade.

According to previous reports, TXA dose regimens for spine surgeries vary widely. A relatively low dose of TXA has been reported to reduce only postoperative blood loss. Tsutsumimoto et al. ${ }^{12}$ reported a study of TXA use in cervical laminoplasty in which study participants received a relatively low dose $(15 \mathrm{mg} / \mathrm{kg})$ of TXA. Postoperative blood loss in the TXA group was reduced by $26 \%$ as compared with the control group. Wang et al..$^{13}$ reported
TABLE 3. Intraoperative and postoperative blood loss

\begin{tabular}{lccr}
\hline \multicolumn{1}{c}{ Variable } & Control Group & TXA Group & p Value \\
\hline Op time (mins) & $192 \pm 47$ & $180 \pm 31$ & $0.27^{*}$ \\
\hline Intraop blood loss $(\mathrm{ml})$ & $415 \pm 247$ & $253 \pm 151$ & $<0.01 \dagger$ \\
\hline Postop blood loss $(\mathrm{ml})$ & $668 \pm 288$ & $321 \pm 123$ & $<0.01 \dagger$ \\
\hline Total blood loss $(\mathrm{ml})$ & $1080 \pm 407$ & $574 \pm 225$ & $<0.01 \dagger$ \\
\hline
\end{tabular}

Data presented as means \pm standard deviations unless otherwise indicated.

* Welch's t-test.

$\dagger$ Mann-Whitney U-test.

a study of TXA use in patients undergoing L2-S1 pedicle screw fixation combined with L4-S1 PLIF who received a relatively low dose $(15 \mathrm{mg} / \mathrm{kg})$ of TXA. Postoperative blood loss in the TXA group was reduced by $13 \%$ compared with the control group. However, in these studies, intraoperative blood loss in the TXA group was not statistically different from the control group.

In contrast, relatively high-dose TXA has been reported to reduce both intraoperative and postoperative blood loss. Elwatidy et al. ${ }^{3}$ reported a study of TXA use in various spine surgeries among various patients who received a relatively high dose of TXA: $2000 \mathrm{mg}$ for adults or $30 \mathrm{mg} / \mathrm{kg}$ for children, followed by continuous infusion of $100 \mathrm{mg} /$ $\mathrm{hr}$ for adults or $1 \mathrm{mg} / \mathrm{kg} / \mathrm{hr}$ for children during surgery, and for 5 hours after the operation. Intraoperative blood loss in the TXA groups was reduced by $49 \%$ and postoperative blood loss in the TXA group was reduced by $55 \%$ compared with the control group. The patient population in their report was mixed: the ages of the patients ranged from children to elderly people, diseases varied, and patients underwent various types of surgical procedures.

Previous studies had limitations in terms of different TXA dose regimens, different levels and numbers of fused

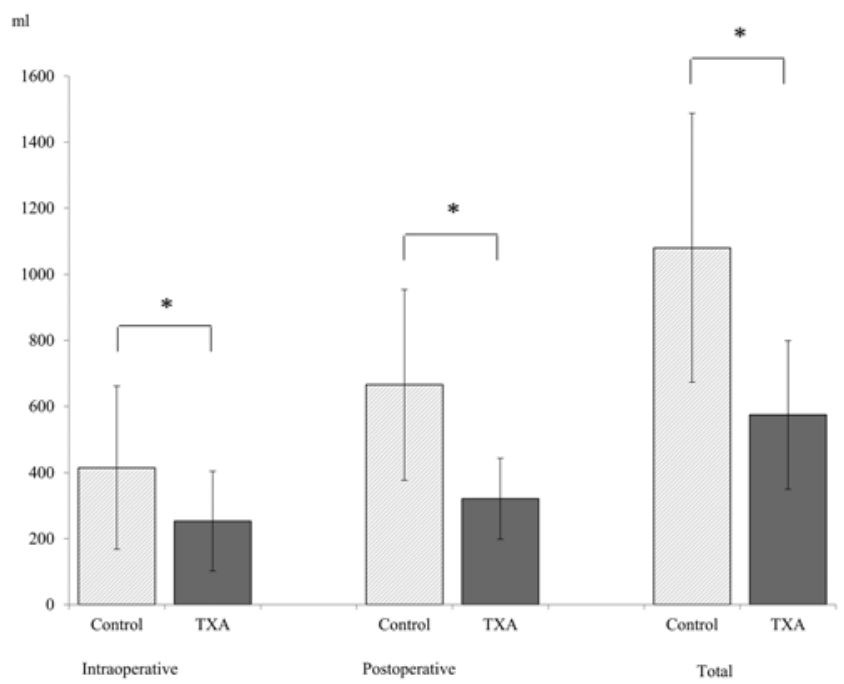

FIG. 1. Bar graph showing intraoperative and postoperative blood loss. The TXA group demonstrated significantly less intraoperative blood loss compared with the control group ( $p<0.01)$. The TXA group also had significantly less postoperative blood loss over 40 hours compared with the control group $(p<0.01)$. Total blood loss in the TXA group was significantly lower than that in the control group. ${ }^{*} p<0.05$. 


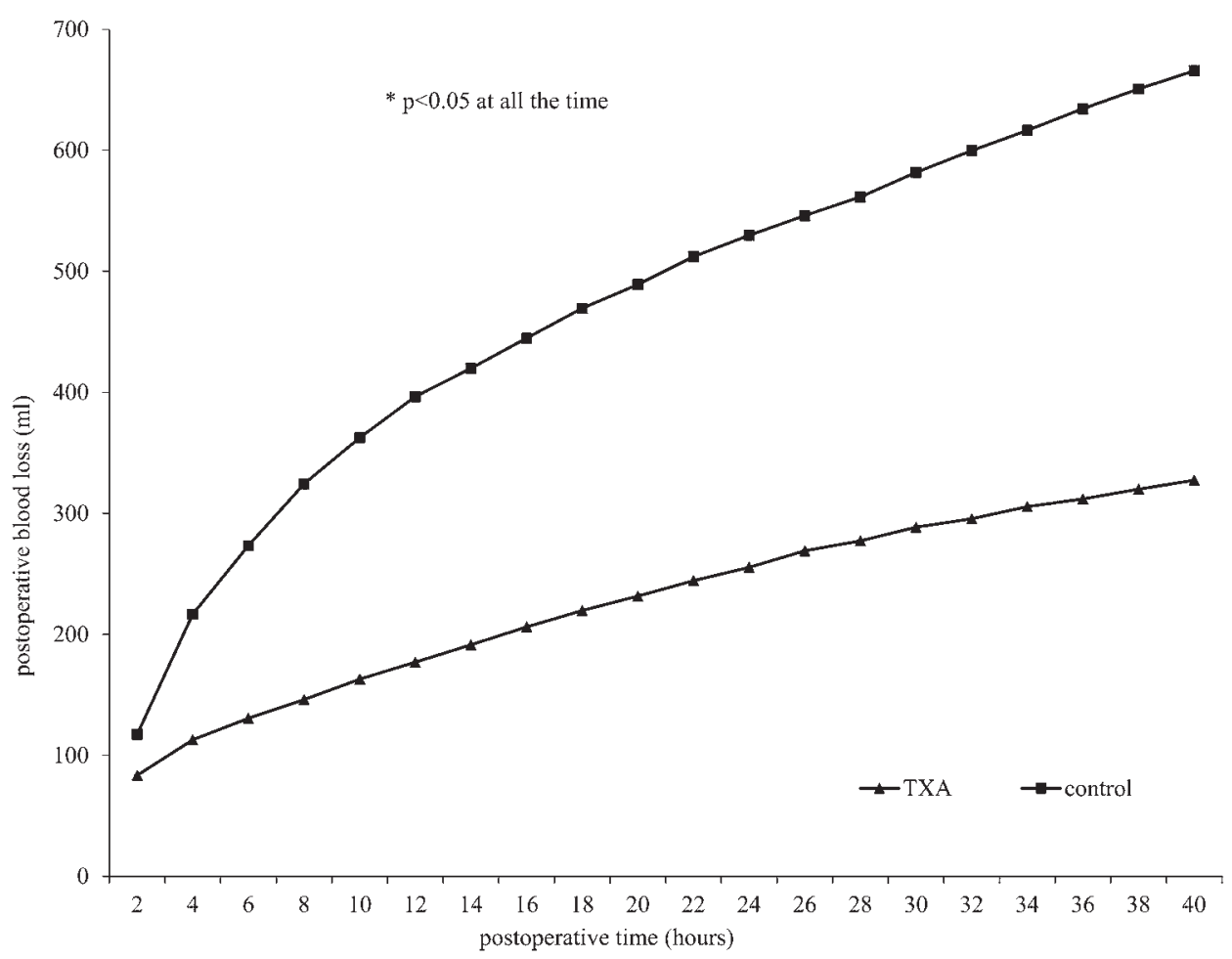

FIG. 2. Line graph showing the time course of postoperative blood loss. From 2 hours to 40 hours, postoperative blood loss in the TXA group was consistently significantly lower than in the control group.

segments, and different surgical techniques. The present study included only patients with surgery performed at the same level and with the same fusion technique. The mean age of each group was likewise almost the same as at primary surgery, so various biases such as age and technical factors could be excluded; thus, the results were very precise.

Total blood loss of $1080 \mathrm{ml}$ in the control group cannot be regarded as a small amount for elderly patients. An intra- and postoperative blood loss negatively affects patient outcomes in various ways. Therefore, surgeons should make the best effort to decrease both intraoperative and postoperative blood loss.

In the present study, the total amount of blood loss could be reduced by $46 \%$ by administration of high-dose TXA during and after PLIF without causing any side effects. The lower the perioperative blood loss, the more advantages for the patients. Less perioperative blood loss contributes to a safe surgery, quick recovery, and patient satisfaction. Administration of high-dose TXA to minimize perioperative blood loss has clinical importance and we strongly recommend spine surgeons use TXA during and after PLIF.

TABLE 4. Hemoglobin and platelet levels on postoperative Day 1

\begin{tabular}{lccc}
\hline \multicolumn{1}{c}{ Variable } & Control Group & TXA Group & p Value $^{*}$ \\
\hline $\mathrm{Hb}(\mathrm{g} / \mathrm{dl})$ & $10.6 \pm 1.5$ & $11.5 \pm 1.3$ & $<0.05$ \\
\hline $\mathrm{PLTs}\left(10^{\circ} / \mathrm{L}\right)$ & $16.0 \pm 3.5$ & $19.0 \pm 4.8$ & $<0.01$ \\
\hline
\end{tabular}

Data presented as means \pm standard deviations unless otherwise indicated.

* Study groups compared using the Student t-test.
The present study has several limitations. First, this is not a double-blind randomized control trial. However, the dose of TXA, surgical techniques, and number of fused segments were strictly limited to make the present study an extremely precise case-control trial. Second, because of the low incidence of perioperative complications, the number of patients may not be enough to examine complications. Further research is required to examine the incidence of complications. The findings of this study should facilitate further research on TXA use in PLIF to determine optimal dose regimens and to survey perioperative complications.

\section{Conclusions}

This study showed that administration of high-dose TXA during and after single-level PLIF could reduce both intraoperative and postoperative blood loss without causing any side effects.

\section{References}

1. Cheriyan T, Maier SP II, Bianco K, Slobodyanyuk K, Rattenni RN, Lafage V, et al: Efficacy of tranexamic acid on surgical bleeding in spine surgery: a meta-analysis. Spine J 15:752-761, 2015

2. Douketis JD, Berger PB, Dunn AS, Jaffer AK, Spyropoulos AC, Becker RC, et al: The perioperative management of antithrombotic therapy: American College of Chest Physicians Evidence-Based Clinical Practice Guidelines (8th Edition). Chest 133 (6 Suppl):299S-339S, 2008

3. Elwatidy S, Jamjoom Z, Elgamal E, Zakaria A, Turkistani A, El-Dawlatly A: Efficacy and safety of prophylactic large dose of tranexamic acid in spine surgery: a prospective, random- 
ized, double-blind, placebo-controlled study. Spine (Phila Pa 1976) 33:2577-2580, 2008

4. Griffiths NJ: Factors affecting the fibrinolytic response to surgery. Ann R Coll Surg Engl 61:12-16, 1979

5. Hardy JF, Desroches J: Natural and synthetic antifibrinolytics in cardiac surgery. Can J Anaesth 39:353-365, 1992

6. Li ZJ, Fu X, Xing D, Zhang HF, Zang JC, Ma XL: Is tranexamic acid effective and safe in spinal surgery? A meta-analysis of randomized controlled trials. Eur Spine J 22:1950-1957, 2013

7. Neilipovitz DT: Tranexamic acid for major spinal surgery. Eur Spine J 13 (Suppl 1):S62-S65, 2004

8. Okuda S, Oda T, Miyauchi A, Haku T, Yamamoto T, Iwasaki M: Surgical outcomes of posterior lumbar interbody fusion in elderly patients. Surgical technique. J Bone Joint Surg Am 89 (Suppl 2 Pt 2):310-320, 2007

9. Pannell WC, Savin DD, Scott TP, Wang JC, Daubs MD: Trends in the surgical treatment of lumbar spine disease in the United States. Spine J 15:1719-1727, 2015

10. Pilbrant A, Schannong M, Vessman J: Pharmacokinetics and bioavailability of tranexamic acid. Eur J Clin Pharmacol 20:65-72, 1981

11. Tanaka N, Sakahashi H, Sato E, Hirose K, Ishima T, Ishii S: Timing of the administration of tranexamic acid for maximum reduction in blood loss in arthroplasty of the knee. $\mathbf{J}$ Bone Joint Surg Br 83:702-705, 2001

12. Tsutsumimoto T, Shimogata M, Ohta H, Yui M, Yoda I, Misawa $\mathrm{H}$ : Tranexamic acid reduces perioperative blood loss in cervical laminoplasty: a prospective randomized study. Spine (Phila Pa 1976) 36:1913-1918, 2011

13. Wang Q, Liu J, Fan R, Chen Y, Yu H, Bi Y, et al: Tranexamic acid reduces postoperative blood loss of degenerative lumbar instability with stenosis in posterior approach lumbar sur- gery: a randomized controlled trial. Eur Spine J 22:20352038, 2013

14. Yang B, Li H, Wang D, He X, Zhang C, Yang P: Systematic review and meta-analysis of perioperative intravenous tranexamic acid use in spinal surgery. PLoS One 8:e55436, 2013

15. Yoshihara H, Yoneoka D: National trends in the surgical treatment for lumbar degenerative disc disease: United States, 2000 to 2009. Spine J 15:265-271, 2015

\section{Disclosures}

The authors report no conflict of interest concerning the materials or methods used in this study or the findings specified in this paper.

\section{Author Contributions}

Conception and design: all authors. Acquisition of data: Kushioka, Yamashita. Analysis and interpretation of data: Kushioka, Yamashita, Iwasaki. Drafting the article: Kushioka, Yamashita, Okuda, Iwasaki. Critically revising the article: all authors. Reviewed submitted version of manuscript: all authors. Approved the final version of the manuscript on behalf of all authors: Kushioka. Statistical analysis: Kushioka, Yamashita. Administrative/ technical/material support: all authors. Study supervision: all authors.

\section{Correspondence}

Junichi Kushioka, Department of Orthopaedic Surgery, Osaka University Graduate School of Medicine, 2-2, Yamadaoka, Suita, Osaka 565-0871, Japan. email: greenday-buzz.ly@hotmail.co.jp. 